\title{
High resolution magnetic force microscopy study of e-beam lithography patterned Co/Pt nanodots
}

\author{
B. D. Belle and F. Schedin \\ School of Computer Science, The University of Manchester, Oxford Road, Manchester M13 9PL, \\ United Kingdom
}

\author{
N. Pilet and T. V. Ashworth \\ Institut fuer Physik der Universitaet Basel, Klingelbergstrasse 82, Basel 4056 Switzerland \\ E. W. Hill and P. W. Nutter \\ School of Computer Science, The University of Manchester, Oxford Road, Manchester M13 9PL, \\ United Kingdom
}

H. J. Hug

EMPA (Swiss federal materials testing centre) Duebendorf, Ueberlandstrasse 129, Duebendorf CH-8600, Switzerland

\author{
J. J. Miles ${ }^{a)}$ \\ School of Computer Science, The University of Manchester, Oxford Road, Manchester M13 9PL, \\ United Kingdom
}

(Presented on 9 January 2007; received 31 October 2006; accepted 11 January 2007; published online 7 May 2007)

E-beam lithography has been used to pattern a continuous $\mathrm{Co} / \mathrm{Pt}$ multilayer film with perpendicular anisotropy into circular islands of various sizes down to $25 \mathrm{~nm}$ diameter on a $60 \mathrm{~nm}$ pitch. High resolution magnetic force microscopy with in situ applied field has been used to directly determine the switching field distribution (SFD) and hysteresis loop of the islands. For the smallest islands, the coercivity is reduced and the width of the SFD is increased, indicating that they would not be well suited to data storage applications. (C) 2007 American Institute of Physics.

[DOI: $10.1063 / 1.2713429]$

\section{INTRODUCTION}

The annual increase in the areal density of hard disk drives has mainly been achieved by scaling, which requires a reduction in grain sizes in order to achieve an acceptable signal to noise ratio (SNR). If grain volumes are reduced too far, their thermal energies become comparable to the anisotropy energy of the grains and can induce random reversals in the magnetization. ${ }^{1}$ Coupled with the requirement of a reasonable number of grains per bit to attain satisfactory SNR, this phenomenon imposes a lower limit to the size of bits, known as the superparamagnetic limit. ${ }^{2,3}$ The fabrication of single domain islands of continuous thin film magnetic media is one method that has been suggested as a possible route to higher density magnetic data storage. ${ }^{4}$

Single domain islands in which the easy axis of magnetization lies perpendicular to the plane and is derived from interface or crystalline anisotropy rather than shape have been proposed for patterned media. ${ }^{3}$ Materials of this nature can be made thin, and the resulting islands could be used with emerging perpendicular recording technology. $\mathrm{Co} / \mathrm{Pt}$ multilayers are therefore good candidates for patterning because of their strong interfacial perpendicular anisotropy, large coercivities, and high squareness. ${ }^{5}$

There have been several studies of the patterning of $\mathrm{Co} / \mathrm{Pt}$ and similar materials using different techniques including: deposition onto patterned resist, ${ }^{6}$ ion beam pattern-

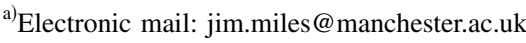

ing using stencil masks, ${ }^{7}$ focused ion beam patterning, ${ }^{8}$ and the deposition onto prepatterned substrates. ${ }^{9}$ The latter technique has produced the best results, with $30 \mathrm{~nm}$ diameter magnetic islands with a periodicity of $60 \mathrm{~nm}$. It does, however, suffer from the drawback of sidewall deposition which could introduce coupling between the dots and the trench.

Preliminary studies of write errors in patterned media indicate that to attain acceptable error rates the switching field distribution (SFD) of the islands will need to be very narrow, and therefore evaluation of the SFD of practical islands is of great interest. ${ }^{10}$ Recent developments in high resolution magnetic force microscopy (HR-MFM) enable a lateral resolution below $10 \mathrm{~nm}$, which allows imaging of the magnetization of $25 \mathrm{~nm}$ nanodots. In this paper, we present the direct measurement of the SFD for a number of island arrays ranging from 25 to $100 \mathrm{~nm}$ fabricated by patterning predeposited $\mathrm{Co} / \mathrm{Pt}$ multilayers using an e-beam lithographically defined hard mask and ion milling for pattern transfer.

\section{METHOD}

Thin film $\mathrm{Co} / \mathrm{Pt}$ multilayers, with a $10 \mathrm{~nm} \mathrm{Pt}$ $+(0.4 \mathrm{~nm} \mathrm{Co}+1 \mathrm{~nm} \mathrm{Pt})_{15}$ structure have been deposited onto $\mathrm{Si}$ substrates using e-beam evaporation at $200^{\circ} \mathrm{C}$. The pressure during deposition was $(1.0-2.1) \times 10^{-7} \mathrm{mbar}$ and the resulting film's coercivity was $200 \mathrm{mT}$. Resist poly methyl methacrylate (PMMA) was then spun onto the multilayers and patterned by direct e-beam lithography at $20 \mathrm{keV}$ using a 


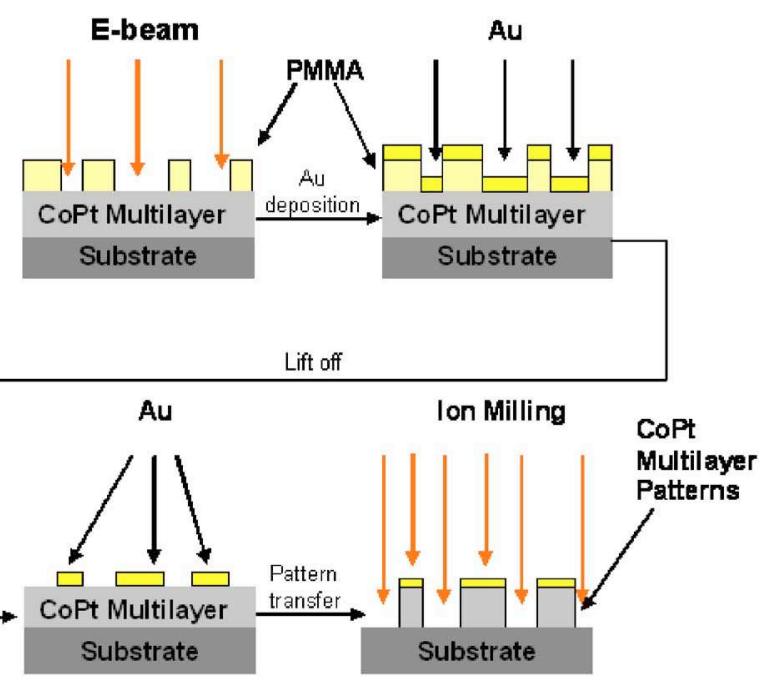

FIG. 1. (Color online) Schematic diagram of the four principal stages in the island fabrication process.

LEO 1530 Gemini field-emission gun (FEG) scanning electron microscopy (SEM) and Raith Elphy Plus lithography system with a laser interferometer stage. After development, e-beam evaporation was then used to deposit $65 \mathrm{~nm}$ of Au. The Au layer from the unexposed regions was removed by lift-off leaving Au nanopillars which were used as a hard mask to transfer the pattern into the magnetic layer using $\mathrm{Ar}^{+}$ milling at $2 \mathrm{keV}$ and $18 \mathrm{~mA}$. The patterning process is illustrated in Fig. 1. Islands ranging from $25 \mathrm{~nm}$ with a center to center separation of $60 \mathrm{~nm}$ up to $500 \mathrm{~nm}$ with a $1000 \mathrm{~nm}$ separation were fabricated, with the smaller islands illustrated in Fig. 2. The resolution of this technique is comparable to that of Ref. 9.

The MFM images were taken at $11 \mathrm{~K}$ using a home built scanning force microscope operating at a base pressure of $1.0 \times 10^{-10}$ mbar. $^{11}$ The microscope is located within a superconducting magnet capable of applying fields up to $7 \mathrm{~T}$ perpendicular to the sample surface allowing for high resolution in situ observation of the magnetic structure at different fields applied along the surface normal. The instrument was operated in the dynamic mode at a constant average height of $12 \mathrm{~nm}$. All images presented in this article were taken at the same tip sample distance and with the same tip
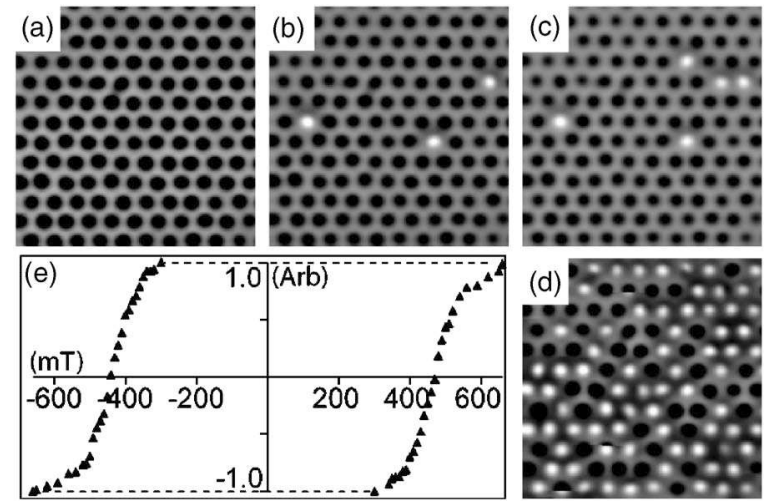

FIG. 3. The hysteresis loop measured in the HR-MFM, showing images at (a) remanance, (b) $-620 \mathrm{mT}$, (c) $-590 \mathrm{mT}$, and (d) coercivity $(-430 \mathrm{mT})$. Images (c) and (b) are one field step $(30 \mathrm{mT})$ apart and show that two islands have a switching field between -590 and $-620 \mathrm{mT}$. In (b) some islands appear to have two domains. These islands were switched by the tip field during imaging.

so as to enable a reliable comparison between images at different fields. Under these conditions the switching field of each dot can be observed and thus the SFD of the array can be determined. ${ }^{12}$

The nanodot array was imaged by scanning a Team Nanotec $\mathrm{GmbH}$ silicon cantilever with a $4 \mathrm{~nm}$ Co coated oxidized tip at a constant average height above the surface, ${ }^{13}$ sufficiently high so as not to have a significant force component from the surface topography. The field generated by the tip was calculated, using the method described by van Schendel et al. ${ }^{14}$ to be $8 \mathrm{mT}$ at the tip apex, falling to $5 \mathrm{mT}$ at $5 \mathrm{~nm}$ below the tip and $3 \mathrm{mT}$ at the scanning height of $12 \mathrm{~nm}$ from the tip. The magnetic contrast in the image enables the determination of the magnetization direction of each dot individually, an example being shown in Fig. 3. The dots exhibit perpendicular anisotropy such that the dot is magnetized either parallel or antiparallel to the tip field, appearing dark or bright, respectively. Using the superconducting magnet, the external field normal to the sample plane was increased incrementally and MFM images were taken at each step of the applied field with the applied field left on throughout the MFM imaging. The switching field of each dot was determined by observing changes in dot magnetization direction between images applied at consecutive field

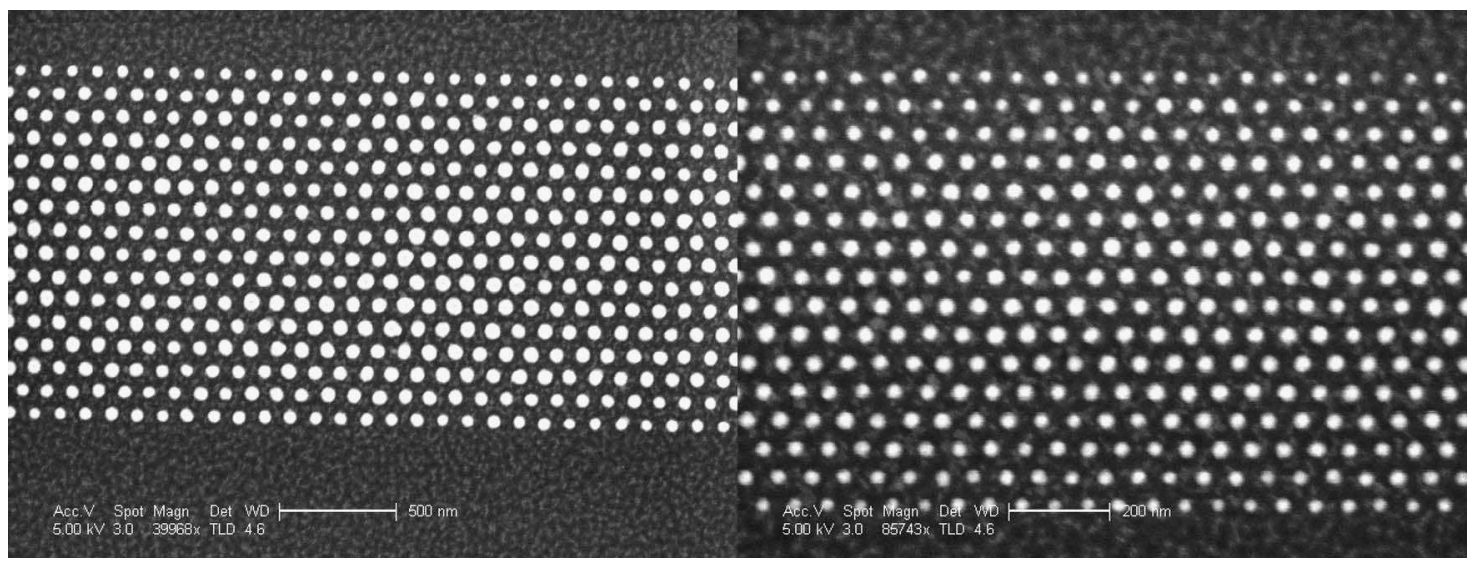

FIG. 2. SEM images of island patterns, showing $50 \mathrm{~nm}$ islands on a $100 \mathrm{~nm}$ pitch (left) and $25 \mathrm{~nm}$ islands on a $60 \mathrm{~nm}$ pitch (right). The areal density of the $25 \mathrm{~nm}$ islands is approximately $210 \mathrm{Gbit} / \mathrm{in}^{2}$. 

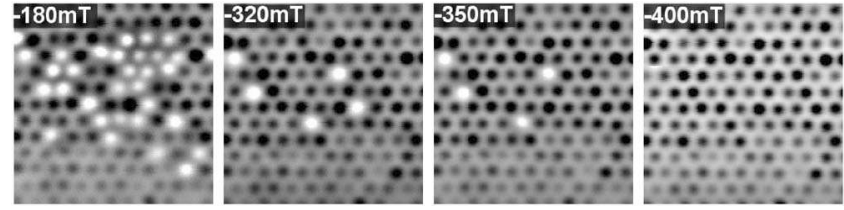

FIG. 4. MFM images of $25 \mathrm{~nm}$ dots at various field steps. The slight blurring at the top and bottom of the images is due to bowing of the substrate and the constant height mode of the instrument.

steps. The SFDs of patterned media with islands of 25,50 , and $100 \mathrm{~nm}$ and spacings of 60,100 , and $200 \mathrm{~nm}$, respectively, were evaluated directly by this method, and their hysteresis loops were then computed by integration of the SFDs. An example hysteresis loop (50 $\mathrm{nm}$ islands) is shown in Fig. 3 , which also shows MFM images of the islands at various stages between saturation and coercivity. In particular, Figs. 3(b) and 3(c) show two images at consecutive fields, showing that it is simple to determine that two out of five dots have switched in that field step. Figure 4 shows MFM images of $25 \mathrm{~nm}$ islands at various field steps. The SFDs of the three samples are shown in Fig. 5. The demagnetizing fields due to the islands were calculated by considering the islands to be arrays of perfect dipoles, and the maximum demagnetizing field computed, which occurred in the saturated state, was $12.5 \mathrm{mT}(125 \mathrm{Oe})$. The computed demagnetizing fields were used to deshear the hysteresis loops before calculating the coercivities and switching field distributions by leastsquares fitting a Gaussian cumulative distribution function (cdf) to the experimental cdf.

In some images islands can be seen to be flipped by the tip as it passes over them, even though the maximum tip field is only $3 \mathrm{mT}$ ( $30 \mathrm{Oe}$ ) at the scanning height of $12 \mathrm{~nm}$ above the top of the islands. This effect could be avoided by scanning the images in zero field, thereby computing remanence loops, ${ }^{15,16}$ but as the tip field contributes an error of only $\sim 3 \mathrm{mT}(30 \mathrm{Oe})$ to the measured hysteresis loops and the loops were measured at field increments of $10 \mathrm{mT}$ this process was not thought to be necessary, and would have been excessively time consuming with the slow field ramp time of the superconducting magnet. Each major loop presented in this paper took $12 \mathrm{~h}$ of continuous measurement.

\section{DISCUSSION AND CONCLUSIONS}

The loops of the 100 and $50 \mathrm{~nm}$ diameter islands are remarkably similar, with average switching fields (coercivities) of 4.4 and $4.5 \mathrm{kOe}$, and standard deviations of 570 and 480 Oe $(13 \%$ and $11 \%)$ for the 50 and $100 \mathrm{~nm}$ islands, respectively, showing no evidence that coercivity increases as size reduces, as was observed by Terris et al. ${ }^{17}$ The $25 \mathrm{~nm}$ islands show a reduced coercivity of $1.4 \mathrm{kOe}$ and a larger standard deviation of 640 Oe $(45 \%)$, which would give rise to a significantly increased write error rate in a practical storage system. This change could be due to the reduced thermal stability of the lower volume islands, but since the experiments were performed at $11 \mathrm{~K}$ and the anisotropy of undamaged $\mathrm{Co} / \mathrm{Pt}$ multilayers is high, it seems much more likely that this is due to edge damage during ion milling. This could take various forms: damage to the interfaces of the multilayer, reducing anisotropy, local irregularities in shape

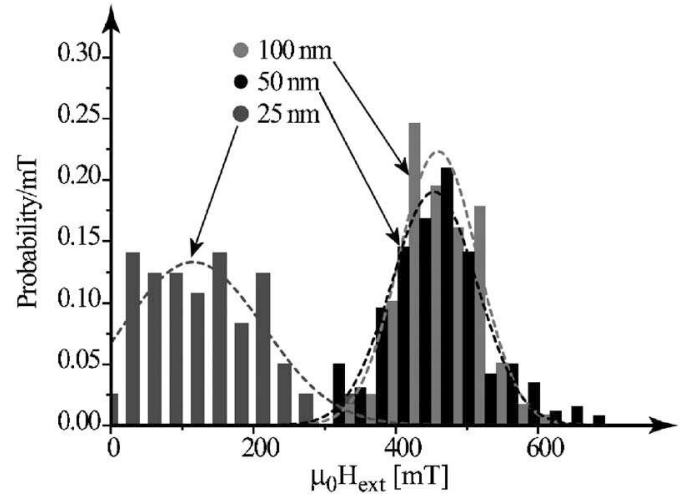

FIG. 5. SFDs of the three samples measured by HR-MFM.

and edge definitions giving rise to nucleation sites, or redeposition of milled material onto the outer edge of the islands. Redeposited material would not be multilayered, and so would have a significantly reduced anisotropy, reducing the switching field of the islands. One possibility would be that the resulting structure with a hard magnetic multilayer core and a soft magnetic outer would have a higher thermal stability than its reduced switching field would suggest in the manner of exchange spring media. ${ }^{18}$ Further measurements at different temperatures are required to correctly determine the reversal processes in these islands and their thermal stability. One island reverses in zero field, suggesting that its switching field is lower than the peak demagnetizing field of $12.5 \mathrm{mT}(125 \mathrm{Oe})$.

In conclusion, we have fabricated magnetic islands as small as $25 \mathrm{~nm}$ diameter with a periodicity of $60 \mathrm{~nm}$. We have demonstrated that the SFD of these arrays can be directly determined by HR-MFM. Decreasing the island diameter below $50 \mathrm{~nm}$ reduces the coercivity and increases the switching field distribution to the detriment of their archival storage potential.

${ }^{1}$ D. Weller and A. Moser, IEEE Trans. Magn. 35, 4423 (1999).

${ }^{2}$ R. M. H. New, R. F. W. Pease, and R. L. White, J. Vac. Sci. Technol. B 12, 1396 (1994).

${ }^{3}$ B. D. Terris and T. Thomson, J. Phys. D 38, R199 (2005).

${ }^{4}$ A. Moser, K. Takano, D. T. Margulies, M. Albrecht, Y. Sonobe, Y. Ikeda, S. Sun, and E. E. Fullerton, J. Phys. D 35, R157 (2002).

${ }^{5}$ C. J. Lin, G. L. Gorman, C. H. Lee, R. F. C. Farrow, E. E. Marinero, H. V. Do, and H. Notarys, J. Magn. Magn. Mater. 93, 194 (1991).

${ }^{6} \mathrm{M}$. Thielen, S. Kirsch, H. Weinforth, A. Carl, and E. F. Wassermann, IEEE Trans. Magn. 34, 1009 (1998).

${ }^{7}$ B. D. Terris, L. Folks, D. Weller, J. E. E. Baglin, A. J. Kellock, H. Rothuizen, and P. Vettiger, Appl. Phys. Lett. 75, 403 (1999).

${ }^{8}$ C. T. Rettner, M. E. Best, and B. D. Terris, IEEE Trans. Magn. 37, 1649 (2001).

${ }^{9}$ G. Hu et al., J. Appl. Phys. 95, 7013 (2004).

${ }^{10} \mathrm{H}$. J. Richter et al., IEEE Trans. Magn. 42, 2255 (2006).

${ }^{11}$ H. J. Hug, B. Stiefel, P. J. A. van Schendel, A. Moser, S. Martin, and H. J. Guntherodt, Rev. Sci. Instrum. 70, 3625 (1999).

${ }^{12}$ N. Pilet, T. V. Ashworth, M. A. Marioni, H. J. Hug, K. Zhang, and J. P. Lieb, J. Magn. Magn. Mater. (in press).

${ }^{13}$ N. Pilet, Ph.D. thesis, University of Basel, 2006.

${ }^{14}$ P. J. A. van Schendel, H. J. Hug, B. Stiefel, S. Martin, and H. Guntherodt, J. Appl. Phys. 88, 435 (2000).

${ }^{15}$ X. Zhu, P. Grutter, V. Metlushko, and B. Ilic, Phys. Rev. B 66, 024423 (2002).

${ }^{16}$ X. Zhu, P. Grutter, V. Metlushko, Y. Hao, F. J. Castano, C. A. Ross, B. Ilic, and H. I. Smith, J. Appl. Phys. 93, 8540 (2003).

${ }^{17}$ B. D. Terris et al., IEEE Trans. Magn. 41, 2822 (2005).

${ }^{18}$ D. Suess et al., Appl. Phys. Lett. 87, 12504 (2005). 